\title{
Expression of transcription factors involved with dehydration in contrasting rice genotypes submitted to different levels of soil moisture
}

\author{
P.A. Auler ${ }^{1}$, M.N. do Amaral ${ }^{1}$, T. Rossatto ${ }^{1}$, I.L. Vighi ${ }^{1}$, L.C. Benitez ${ }^{2}$, \\ L.C. da Maia $^{3}$ and E.J.B. Braga ${ }^{1}$ \\ ${ }^{1}$ Departamento de Botânica, Instituto de Biologia, Universidade Federal de \\ Pelotas, Pelotas, RS, Brasil \\ ${ }^{2}$ Unidade Acadêmica de Ciências Exatas e Natureza, Universidade Federal \\ de Campina Grande, Campus Cajazeiras, Cajazeiras, PB, Brasil \\ ${ }^{3}$ Centro de Criação de Plantas, Universidade Federal de Pelotas, Pelotas, RS, \\ Brasil
}

Corresponding author: P.A. Auler

E-mail:pri_auler@hotmail.com

Genet. Mol. Res. 18 (1): gmr18247

Received January 15, 2019

Accepted February 21, 2019

Published March 31, 2019

DOI http://dx.doi.org/10.4238/gmr18247

\begin{abstract}
Plant tolerance to abiotic stresses, such as water deficit, is triggered by complex multicomponent signaling pathways. One of the plant responses to stress conditions is expression of a large number of genes whose products are involved in various adaptive functions. Transcription factors (TFs) are important regulators of gene expression, modulating the transcription initiation rate of target genes; they are critical components in signal transduction in response to abiotic stress. Our hypothesis is that genotypes with contrasting tolerance signal water deficit through TFs (10 genes of the DREB family and bZIP) with differing degrees of expression. We believe that these genes will have greater expression in the flooded genotype because drought is an atypical condition in these plants. We tested two rice (Oryza sativa) genotypes: BRS Querência (flooded) and AN Cambará (dryland). When they reached stage V5, the plants were submitted to water deficit, at different levels of soil moisture, $20 \%, 10 \%$ and recovery. In general, we observed that expression varies according to the soil moisture and the
\end{abstract}


genotype. In addition, the coexpression analysis generated seven cluster interactions with several genes. Our hypothesis was confirmed in part; the contrasting genotypes gave different degrees of expression; however, we ca not affirm that the flooded genotype has more significant responses in dry soil because there were variations in expression demonstrating oscillation in metabolism, and the data do not allow us to associate expression with greater drought tolerance.

Key words: Coexpression; Drought; Networks; Oryza sativa; RiceFrend

\section{INTRODUCTION}

In Brazil, there are two large ecosystems for rice cultivation, known as flooded, where rice is cultivated with controlled flood irrigation, and dryland, which is mainly dependent on rainfall (Guimarães et al., 2006); the latter option often results in stress to the plant due to water deficit. Various studies have been conducted to increase the efficiency of the rice cultivation system under conditions of water deficit (Gowda et al., 2011; Serraj et al., 2011; Shi et al., 2012). The effects of this stress varies, depending on many factors, such as intensity and duration of the drought, the genotype and the plant growth phase (Foley et al., 2011).

Under ideal conditions, plants are in homeostasis, but when subjected to stress situations, they initially enter an alarm/alert phase, where there is intense signaling, first at the electrical/molecular level, so that plant defense mechanisms are activated (Cabane et al., 2012). The intensity of this signaling will depend on the severity of the stress and the time of exposure.

Plant tolerance to abiotic stresses, such as water deficit, is triggered by complex multicomponent signaling pathways to restore cell balance and promote survival (Golldack et al., 2014). One of the plant responses to stress conditions is the expression of a large number of genes whose products may be involved in various adaptive functions (Thomashow, 1999). Among these functions, studies suggest that the regulation of transcription is one of the most important steps for adapting plants to stress conditions (Zhu, 2002; Yamagushi-Shinozaki and Shinozaki, 2006). From functional studies with Arabidopsis thaliana, several genes that are induced by abiotic stresses have been characterized, acting as primers for the perception and the transmission of the signals between the cells (Yamagushi-Shinozaki and Shinozaki, 1993).

Transcription factors (TFs) are important regulators of gene expression. In general, a TF is composed of at least two distinct domains, a DNA binding and an activation/repression domain, which operate together to regulate the different physiological and biochemical processes, modulating the rate of transcription initiation of the genes target (Ptashne, 1988) being critical components in signal transduction in response to abiotic stresses. Plants attribute much of their genome to TFs. More than 1600 genes $(7 \%$ of the genome) were identified in Arabidopsis (Riechmann et al., 2000), revealing the complexity of regulation at the transcriptional level. The genes of the TFs DREB family (dehydration responsive element binding) encode proteins that have a conserved functional domain, known as AP2/EREBP, which plays an important role in the regulation of genes responsive 
to biotic and abiotic stresses (Sakuma et al., 2002). Other genes that are related to environmental stress response and tolerance are those belonging to the ERF family (Ethylene Responsive Factors). The ERF TF family constitutes a broad category of stress responsive genes that are involved in the mediation of abiotic conditions via signaling. Control of regulation by the ERF family and its target genes is fundamental for understanding how plants respond to adverse conditions and how tolerance mechanisms involving this molecular signaling can be achieved, which is an important step in the genetic enhancement (Yamaguchi-Shinozaki and Shinozaki, 1994).

Another important group of TFs involved in stress responses consists of genes that contain the bZIP domain, being one of the largest families found in plants, and present in all eukaryotes. Expression analyzes revealed that 33 members are responsive to drought (Nijhawan et al., 2008). Of these genes, five, $A B F 3$ (Oh et al., 2005), OsbZIP23 (Xiang et al., 2008), OsbZIP46 (Tang et al., 2012), OsbZIP52 (Liu et al., 2012) and OsbZIP72 (Lu et al., 2009) have conferred drought tolerance when overexpressed in rice.

An important way to achieve tolerance to multiple stress conditions is overexpression of transcription factors that control a wide variety of genes in different pathways. Transgenic plants that overexpress DREB transcription factors show greater tolerance to drought, high salinity, heat and freezing (Lata and Prassad, 2011).

Advances in genetic and molecular biology tools have encouraged progress in genetic architecture discovery, but also in the regulatory mechanisms of complex traits (Mochida and Shinozaki, 2011). The development of molecular profiling techniques nowadays allows the acquisition of large data sets of omics such as transcriptomics, proteomics and metabolomics (Serin et al., 2016). While substantial efforts are being made to generate large datasets of information, there is a growing need to develop platforms to integrate such data and derive models that describe the biological interactions in plants. In this context, networks quickly became an attractive approach to manage, display, and contextualize these large datasets (Usadel et al., 2009; Silva et al., 2016). Biological networks are generally classified by the nature of the compounds and the interactions involved. These networks may be derived from various molecular data resulting in, for example, gene expression networks (correlation or co-expression networks), protein-protein interaction (PPI) networks, metabolic networks and signaling networks. These interaction maps provide an attractive framework for studying the organizational structure of complex systems and found many applications in plants (Jiménez-Gómez, 2014).

Therefore, the hypothesis of our study is that genotypes with contrasting tolerance signal the water deficit, through TFs (10 genes of the DREB family and bZIP), in different degrees of expression. We believe that the genes studied will have a greater expression in the flooded genotype because drought is an atypical condition in these plants. We also believe that some genes studied respond more actively to rehydration after a short period of deficit.

The aim of our paper was to evaluate the pattern of TFs expression in two genotypes of rice, BRS Querência (flooded) and AN Cambará (dryland), seeking to identify significant changes that may help in the understanding of signaling when plants are submitted to different moisture soil (CC: field capacity, 20\% and 10\%); and determine if there is difference in these expressions when plants are submitted to water deficit and recovered (rehydrated) after $24 \mathrm{~h}$. To better understand these responses, we analyzed the 
interaction network of these TFs as well as the correlated genes deposited in silico with the aid of the program RiceFrend (Sato et al., 2013).

\section{MATERIAL AND METHODS}

\section{Plant Material and Experimental Conditions}

The experiment was conducted using two rice genotypes: BRS Querência (subspecies indica), which is preferably used in flood soils and using a flooding irrigation system, and genotype AN Cambará (subspecies indica), grown in dry soils, mostly using an aspersion irrigation system, where there is less water availability. Both genotypes have similar life cycles (precocious).

The seeds were disinfected with $1 \%$ hypochlorite, placed to germinate on germitest paper in rolls and maintained in a Biological Organism Development (BOD) growth chamber, with a 16 hour photoperiod of light and $8 \mathrm{~h}$ of dark at $25 \pm 2^{\circ} \mathrm{C}$ for 10 days. After this period, the seedlings were transferred to plastic vases $(8 \mathrm{~L})$, perforated at the base, containing soil collected from the field and properly corrected in accordance with the needs of the crop. In each pot, six seedlings were transplanted and kept in a greenhouse at a temperature of $25 \pm 2{ }^{\circ} \mathrm{C}$ with a relative humidity of $70 \%$ and controlled irrigation, depending on the treatment.

The soil remained in the field capacity (40-45\% soil moisture) until the plants reached the V5 stage (water-sensitive stage), where they were submitted to water deficit, at different levels of soil moisture, being $20 \%$ soil moisture (collection 1), $10 \%$ soil moisture (collection 2) and recovery (collection 3), which consists in reestablishing the ideal water condition (40-45\% soil moisture) by 24 hours. The control treatment was maintained under ideal conditions of humidity. Soil moisture was monitored with a TDR probe (Decagon Devices ${ }^{\circledR}$ ), which determines the relative leaf water content (RWC) by measuring the dielectric constant of the medium using the technology of capacitance/frequency domain.

\section{Relative leaf water content (RWC)}

The RWC was evaluated according to Smart and Bingham (1974).

\section{Total RNA Extraction and cDNA Synthesis}

Total RNA was extracted from $0.1 \mathrm{~g}$ of leaves using the PureLink ${ }^{\circledR}$ Kit (Invitrogen ${ }^{\mathrm{TM}}$ ). For the degradation of contaminating DNAs, the samples were treated with DNAse I (Invitrogen ${ }^{\mathrm{TM}}$ ). The quality and integrity of total RNA were evaluated by $1 \%$ agarose gel electrophoresis and the quantity and purity in NanoDrop ND-1000. Each sample was reverse-transcribed (mRNA) into cDNA, using the commercial SuperScript FirstStrand System for the RT-qPCR kit (Invitrogen ${ }^{\mathrm{TM}}$ ).

\section{RT-qPCR reactions}

The total reaction volume was $12 \mu \mathrm{L}$, of which $6.25 \mu \mathrm{L}$ were of the kit, with SYBR Green fluorophore (Roche), $0.25 \mu \mathrm{L}(10 \mathrm{mM})$ of each (sense and antisense) primer, $1 \mu \mathrm{L}$ of 
cDNA (dilution 1:5, previously defined), and $4.25 \mu \mathrm{L}$ ultra-pure water. The reactions were carried out in the Bio-Rad CFX Real Time Thermal Cycler using the following amplification parameters: $95^{\circ} \mathrm{C}$ for $10 \mathrm{~min}, 40$ cycles of $95^{\circ} \mathrm{C}$ for $15 \mathrm{~s}, 60^{\circ} \mathrm{C}$ for 1 min with insertion of the melting curve from 65 to $95^{\circ} \mathrm{C}$, with $5^{\circ} \mathrm{C}$ increases at each fluorescence measurement. For each biological sample, three technical samples (triplicates) were run. Normalizing genes were used $U B C-E 2$ (ubiquitin conjugated to E2 enzyme) and UBQ5 (Ubiquitin 5), previously tested for the experimental conditions (Auler et al., 2016).

Ten genes coding for transcription factors related to dehydration of the DREB and bZIP family were selected for this study, being: bZIP23 (Os02g0766700), bZIP46 (Os06g0211200), bZIP72 (Os09g0456200), DREB1A (Os09g0522200), DREB1B (Os09g0522000), DREB1C (Os06g0127100), DREB1E (Os02g0676800), DREB1F (Os01g0968800) DREB1G (Os02g0677300) and DREB2A (Os01g0165000). The primers (listed in Table 1) were designed using the sequences deposited in the RAP-DB database and the PerlPrimer v.1.1.21 program. The RT-qPCR data was exported to Excel (Microsoft Excel, 2010) and the graphics generated using the Origin 9.0 program. The relative expression data were calculated according to the method $2^{-\Delta \Delta Q c}$ (Livak and Schmittgen, 2001). The specificity of each set of primers was verified through the dissociation curve (melting curve) and only those with specific amplicons were kept in this study, i.e. with a single peak of dissociation from the strands produced in the RTqPCR (Supplementary 1$)$.

Table 1. Description of the primers used in RT-qPCR reactions in rice seedlings submitted to different levels of soil moisture.

\begin{tabular}{llll}
\hline Gene & Access & Primer Sense 5'-3' & \\
\hline$D R E B 1 A$ & AK105599 & GACGTCCTGAGTGACATGGG & Antisense 5'-3' \\
$D R E B 1 B$ & AK062422 & TCGCACTGAAAAGTGTGGAC & AGTAGCTCCAGAGTGGGACG \\
$D R E B 1 C$ & AY327040 & ACTGATGATCGCGAGTTGGA & AGGAGGGAGAAATCTGGCAC \\
$D R E B 1 E$ & AY1 14110 & ACTTCCCTTGCTACCCGATG & AGGAGGAGCAAAGCTGGTTG \\
$D R E B 1 F$ & AY345234 & AAGCTATGGATGGACTGACATGTAT & GCTCGATGAGCATTCCCTGT \\
$D R E B 2 A$ & AK067313 & AGCCAAGGGGTCCAAGAAAG & CCGTATTTAGTAGGAACTGATGGAG \\
$D R E B 1 G$ & AK106041 & GGCCAGATCGAGCTTATGGG & CACCCACTTACCCCATGTCC \\
$b Z I P 23$ & AK072062 & CGCCAGAGGAAACAGGCATA & ACACTCGACAAGCCTTCACC \\
$b Z I P 46$ & AK103188 & TAATTCGGGCCATCGCATCA & GGTCCAACTTGTCGGCTCAT \\
$b Z I P 72$ & AK065873 & CCCACCCTAGAGACCAGACA & AGCGGAGAAGAGAGGAGTGT \\
$U B C-E 2^{l}$ & AK059694 & CCGTTTGTAGAGCCATAATTGCA & TCTTGGTCCCCATGACTTGC \\
$U B Q 5^{l}$ & AK061988 & ACCACTTCGACCGCCACTACT & AGGTTGCCTGAGTCACAGTTAAGTG \\
\hline
\end{tabular}

${ }^{1}$ Auler et al. (2016)

\section{Coexpression Analysis}

In this study, we used the RiceFREND platform (Sato et al., 2013), which uses data from the Rice Expression Database Database (RiceXPro) to assemble the networks. The RiceXPro is a repository of gene expression profiles derived from microarray analysis of tissues/organs encompassing the entire growth of the rice plant under natural field conditions, rice seedlings treated with various phytohormones, and specific cell types/tissues isolated by laser microdissection (LMD). This database is part of a project on rice transcriptome analysis using microarray technology aimed at characterizing the expression profile of all predicted genes in rice and providing reference information that can be used in functional genomics (Sato et al., 2011). All expression profiles were generated 
using a single microarray platform with probes based on manually curated gene models in RAP-DB and rice full-length cDNA sequence information in KOME database.

\section{Experimental design and statistical analysis}

The experimental design was completely randomized (DIC) in a factorial scheme $(2 \times 4)$ being two genotypes and four different levels of soil moisture (CC, 20\%, 10\% and Rec). Six biological collections were performed. The experimental unit consisted of a vessel containing six plants, each vessel being a biological replicate. The data were submitted to analysis of variance $(P \leq 0.05)$ and the means were compared by the Tukey test at $5 \%$ of error probability, using the software R 3.1.2 (RCore Team, 2013).

\section{RESULTS}

Analyzing the relative water content in the leaves (RWC \%) we can observe that as the water decreases in the soil, the \% of water in the leaves decreases. Associated with this, after $24 \mathrm{~h}$ of rehydration the $\%$ of water in the plants that were under deficit resembles that of the control (always hydrated) in both genotypes. This analysis serves to demonstrate physiologically the water deficit and recovery in the plant leaves (Figure 1).

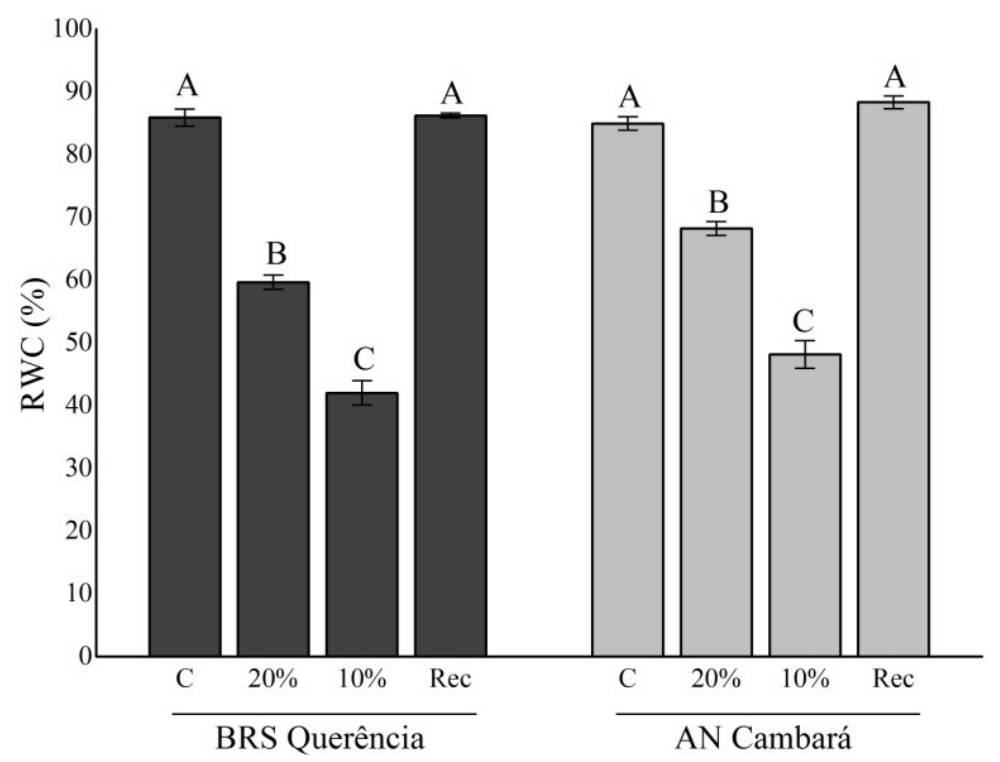

Figure 1. Relative water content in leaves (RWC) in BRS Querência and AN genotypes at different levels of soil moisture (field capacity, 20\%, 10\% and $24 \mathrm{~h}$ of recovery) in the V5 stage. Upper case letters compare stressed plants with their respective controls within each genotype. The genotypes did not differ significantly from each other by the Tukey test at $5 \%$ of error probability.

In relation to the expressions of the TFs studied, we can observe that the expression varies according to the soil moisture and the genotype (Figure 2). For OsbZIP23, in BRS Querência, the expression value at $20 \%(\mathrm{RQ}=3.27)$ was higher than the $10 \%(\mathrm{RQ}=2.63)$ and $\operatorname{Rec}(\mathrm{QR}=2.21)$ (Figure 2a). With this, it is observed that for this gene, except in the 
control, in all the other treatments there was significant difference. When observing OsbZIP46, in BRS Querência there was no difference in expression between the different soil moisture levels, however, they were superior to the control in all treatments. In AN Cambará, at $10 \%$ moisture, the expression of this $\mathrm{TF}$ was approximately $15 \mathrm{X}(\mathrm{RQ}=15.10)$ higher than the control, whereas in $20 \%$ and recovery there was no significant difference between these moisture (Figure 2b). For BRS Querência, the expression of OsbZIP72 differed between treatments when the plants were rehydrated for $24 \mathrm{~h}$, increasing around $6 \mathrm{X}$ $(\mathrm{RQ}=6.16)$. In AN Cambará, when the stress intensified (10\%), the relative expression was superior $(\mathrm{RQ}=2.16)$ to the other treatments (Figure $2 \mathrm{c}$ ).

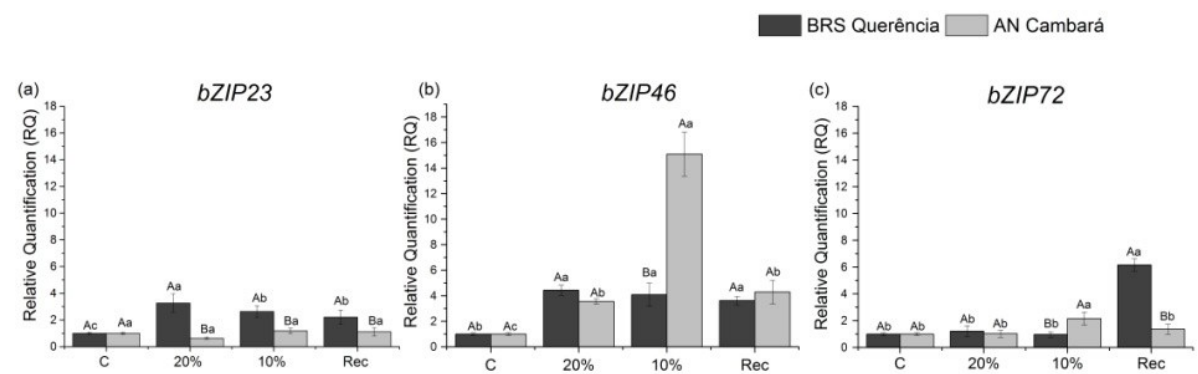

Figure 2. Relative quantification (RQ) of the bZIP family genes (a) bZIP23, (b) bZIP46 and (c) bZIP72 in the rice genotypes, BRS Querência and AN Cambará, at different levels of soil moisture (CC, 20\%, 10\% and Recovery - Rec). Upper case letters compare genotypes within each treatment and lower case compare treatments in the same genotype.

In relation to $O S D R E B 1 A$, the genotypes differed significantly from each other in the treatments of $10 \%$ and Rec, and in AN Cambará at 10\% the expression of this gene was around 3x higher than the control. As for BRS Querência, when the plants were rehydrated $(\mathrm{Rec})$ this gene had a $6 \mathrm{X}$ higher expression than the control plants (Figure 3a).

The expression of $O S D R E B 1 B$ in BRS Querência leaves was statistically different at $20 \%, 10 \%$ and Rec, being the highest value when the plants were rehydrated $(\mathrm{RQ}=4.08)$, followed by $10 \%(\mathrm{RQ}=2.25)$ and $20 \%(\mathrm{RQ}=0.50)$. In $20 \%$, AN Cambará and BRS Querência were similar, because in both the expression value was inferior to the control (Figure 3b).

For OsDREBIC, there was no significant difference between the treatments in BRS Querência, while in AN Cambará, at $10 \%$ soil moisture the expression increased around $5 X(R Q=4.96)$ when compared to the control $(R Q=1.00)$ (Figure $3 \mathrm{c})$.

BRS Querência and AN Cambará had opposite responses in the expression of OsDREBIE, and for the treatments of $20 \%$ and $10 \%$, the flooded genotype had low expression ( $\mathrm{RQ}=0.05$ and $\mathrm{RQ}=0.41$ respectively), lower than the control, and equally to him in the recovery $(\mathrm{RQ}=0.99)$. However, it was observed that in AN Cambará, at $20 \%$ and $10 \%$ of humidity, the expression was superior to the control (RQ $=1.42$ and $\mathrm{RQ}=2.11$ respectively) and lower in the recovery $(\mathrm{RQ}=0.31)$ (Figure $3 \mathrm{~d}$ ).

With OSDREBIF, the expression was superior to the control only in the treatment of $10 \%(\mathrm{RQ}=1.14)$ in BRS Querência and in $20 \%$ and $10 \%$ in AN Cambará 
$(\mathrm{RQ}=1.46$ and $\mathrm{RQ}=1.73$ respectively) (Figure $3 \mathrm{e}$ ). In addition, the expression of OsDREB $1 G$, both in the flooded and dryland genotypes, at $20 \%$ was lower than the control ( $\mathrm{RQ}=0.15$ and $\mathrm{RQ}=0.03$ respectively), whereas in BRS Querência at $10 \%$ and Rec were statistically the same between them $(\mathrm{QR}=1.30$ and $\mathrm{QR}=1.24$ respectively). In AN Cambará, at $10 \%(\mathrm{RQ}=0.95)$ resembled control and in $\mathrm{Rec}$ was lower $(\mathrm{RQ}=$ 0.80) (Figure 3f).

For both genotypes, the expression of OsDREB2A was low at $20 \%(\mathrm{QR}=0.06$ and $\mathrm{QR}=0.02)$, but in the flooded genotype it was higher in $\operatorname{Rec}(\mathrm{QR}=1.55)$ and dryland at $10 \%(\mathrm{QR}=1.62)$ (Figure $3 \mathrm{~g}$ ).

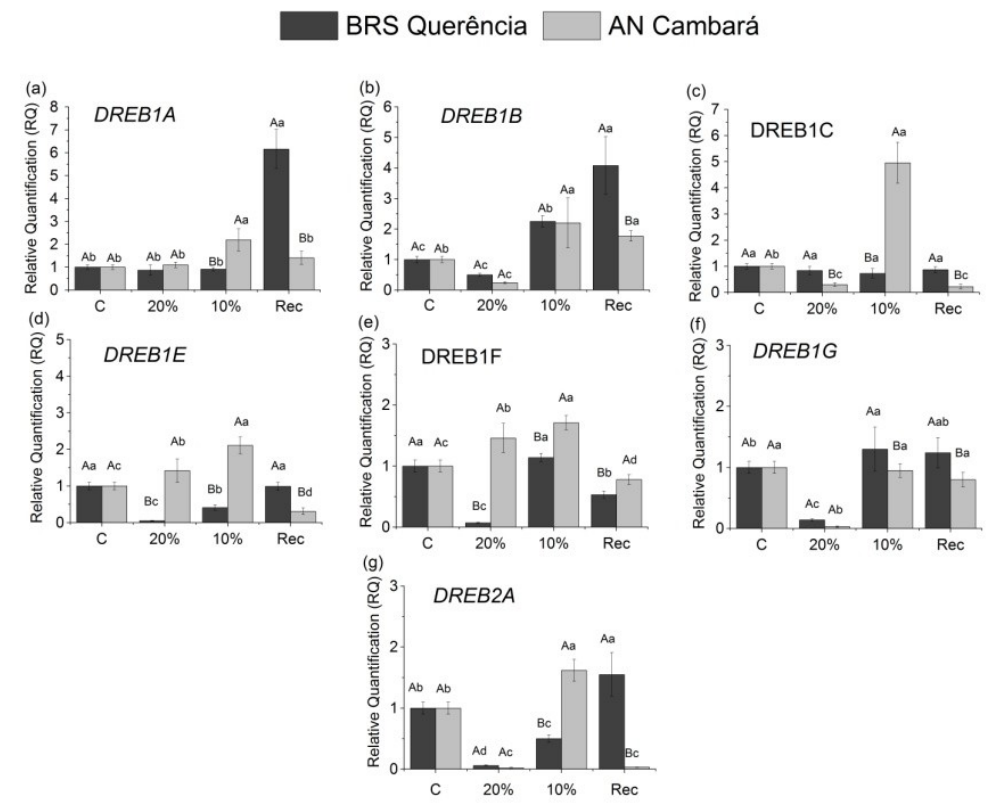

Figure 3. Relative quantification (RQ) of DREB family genes (a) DREB1A, (b) DREB1B, (c) DREB1C, (d) $D R E B 1 E$, (e) $D R E B 1 F$, (f) $D R E B 1 G$ and (g) $D R E B 2 A$ in the rice genotypes, BRS Querência and AN Cambará, at different levels of soil moisture (CC, 20\%, 10\% and Recovery - Rec). Upper case letters compare genotypes within each treatment and lower case compare treatments in the same genotype.

The coexpression analysis generated seven clusters with interaction with several genes, whereas DREB1E (Os02g0676800) did not interact, whereas bZIP23 (Os02g0766700) had a correlation with several genes, showing the different causes of variation (Figure 4). In addition we can observe the different interactions with genes of different routes and with variable functions. We observed the highest correlations between closely related genes, such as Os01g0968800 (DREB1F) X Os05g0162800 (DNA binding domain) (0.85), Os03g0820300 (TF zinc finger) X Os03g0820400 (TFIIIA-type zinc finger protein, transcription activator, abiotic stress tolerance) (0.84) and Os01g0846300 (phosphatase) X Os09g0325700 (phosphatase) (0.82). The complete list of correlation values is found in the Supplementary 2. The information of the routes related to the specific genes is demonstrated in Table 2 . 
Table 2. Identification of genes with metabolic routes according to Kegg, indicated by different colors.

\begin{tabular}{llc}
\hline Kegg Pathway & Locus ID & Specific Pathway \\
\hline Osa01100 Metabolic pathways & Os02g0274100 & Os06g0103300 \\
& Os09g0555500 & \\
Osa00906 Carotenoid Biosynthesis & Os09g0555500 & \\
Osa04650 Natural Killer cell mediated cytotoxicity & Os03g0285800 & \\
Osa00592 Alpha-Linolenic acid metabolism & Os02g0274100 & \\
Osa00350 Tyrosine metabolism & Os06g0103300 & Os04g0224900 \\
Osa00564 Glycerophospholipid metabolism & Os01g0955100 & \\
Osa04626 Plant- pathogen interaction & & \\
\hline
\end{tabular}

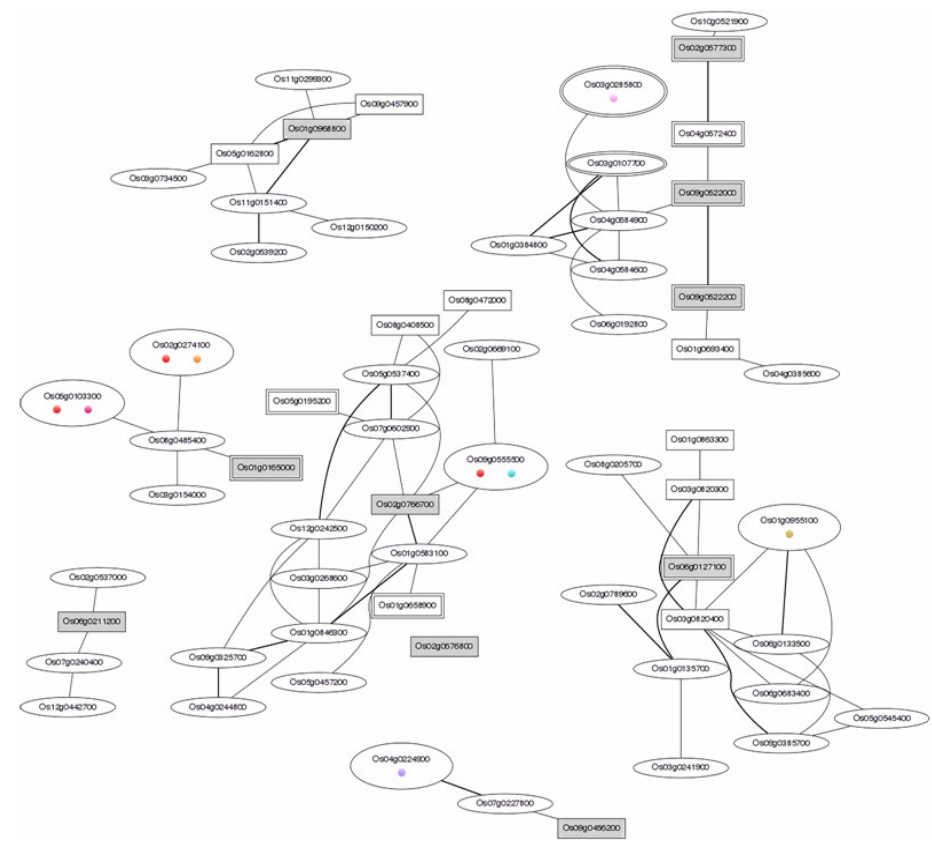

Figure 4. Network graphic with connection between multiple genes. The gray rectangular boxes represent the transcription factors discussed in this study. In the circles are represented the genes correlated to these TF and circles with indication with color, genes related to metabolic routes according to database KEGG. The color intensity of the lines is directly proportional to the level of interaction. The descriptions of all genes, according to the identification of the figure, are in the Supplementary 2, as well as the list of nodes and the interaction values.

\section{DISCUSSION}

The water deficit is characterized as one of the most impacting for most agricultural crops. Allied to this, the need to use less water in the crops has been increased by the changes caused by climate change (Pandey and Shukla et al., 2015). The molecular responses to abiotic stresses interfere with all scales of the organism, including the perception of stress, signal transduction to the cell components, gene expression and then metabolic alterations, which can confer stress tolerance (Agarwal et al., 2006). Thus, genes do not only function in the protection of cells to stress by the production of metabolic proteins, but also in the regulation of responses and signal transduction (Lata and Prasad 2011). The different levels of soil moisture vary the relative water content in the leaves as 
seen in figure 1, characterizing the maintenance of water stress. The RWC was decreasing as the amount of water in the soil decreased, and after the rehydration the control plants were similar, evidencing that the plants had their water reestablishment recovered.

The variation in expression values between treatments and among genotypes shows that for each level of water deficit or after passing through it (recovery) different transcription factors associated with this condition are activated, characterizing a dynamic and particular gene response in the cascade of reactions. According to Seeve et al. (2016), $\mathrm{TF}$ expression levels in corn seedlings submitted to different levels and times of water deficit varied widely depending on the condition and the tissue. The authors believe that these results, taken as standards, are critical for future studies that seek to elucidate the TFs involved in regulating plant responses to stress, since their responses seem to be quite flexible, as we observed in our study.

In studies with $D R E B 1 A$ in peanut under water deficit, there was an increase in expression in this gene only after three days of exposure, but 3-5 days there was a significant increase in mRNA (Bhatnagar-Mathur et al., 2014). In our study, in the BRS Querência genotype, we detected an increase in expression after 24 hours of rehydration $(\mathrm{Rec})$, and may be a gene that also responds to water recovery. We believe that in the AN Cambará genotype it will change the level and the stress time did not trigger response of this gene, in view of its greater tolerance. Plants of Salvia miltiorrhiza transformed overexpressing DREB $1 A$ also demonstrated increased drought tolerance as well as increased bioactive compounds without detecting phenotypic changes (Wei et al., 2016). It has been shown that overexpression of $D R E B 1 A$ and $D R E B 1 E$ in rice contributes to the survival of plants under water deficit (Ito et al., 2006; Chen et al., 2008). In AN Cambará it was observed that the DREBIE TF had superior expression (at 10\%) than the other treatments and that BRS Querência, and may be related to adaptation/tolerance mechanisms. We also believe that this gene has a faster response than the others, being activated at the initial moments of signal perception. Several studies with $D R E B 2 A$ have been used to develop plants with drought tolerance. In Arabidopsis, overexpression of DREB2A resulted in significant tolerance for stresses due to drought and heat (Sakuma et al., 2006a,b). In addition, studies with AtDREB2A homologues in maize (Qin et al., 2007), rice (Dubouzet et al., 2003), sunflower (Almogueva et al., 2009) and wheat (Terashima and Takumi, 2009) demonstrated similar tolerance increase. In our study, in the genotype of the dryland (AN Cambará), at the moment when the plants were under $10 \%$ of water in the soil, DREB2A had its greater expression, being able to be related to the level of tolerance of this genotype against these conditions and in the presence showed expression below the control, demonstrating the difficulty of adaptation.

Wei et al. (2016) found that Salvia miltiorrhiza seedlings overexpressing AtDREB1C had increased survival rates under severe drought conditions when compared to wild plants (WT-control), and differentially expressed genes were mainly related to photosynthesis, signaling, hormones and secondary metabolism. Under soil moisture conditions at $10 \%$, in the AN Cambará genotype, DREB1C had higher expression than the other conditions, probably because it was more adapted to water restrictions and signalized tolerance mechanisms more efficiently. Studies with Arabidopsis and Tobacco transgenics have shown strong evidence that $D R E B 1 B$ is involved in stress responses of biotic and abiotic origin conferring broad spectrum of tolerance (Reddy and Gutha 2008). The overexpression of bZIP72 in maize increases the expression of ABA-inducible genes as 
well as ZIP72 proteins, which act as positive regulators in water deficit tolerance and are candidates for genetic improvement in crop adaptation to drought (Dubey et al., 2016). In plants of the genotype of flooded, that is, more susceptible to dehydration, we observed a high expression of this TF after rehydration, which could be due to a late response of expression, or as a gene activated after a water limitation, promoting the synthesis of compounds in case of another exposure.

The bZIP genes of rice, $b Z I P 23$ and $b Z I P 46$ also belong to the ABF/AREB family and the activation of each of these genes increased drought tolerance through increased ABA response and expression of stress related genes (Xiong et al., 2008). For bZIP23, in all levels of deficits tested, as well as in recovery, the expression of this gene was higher than in the control plants in the BRS Querência genotype, and it may be related to the greater sensitivity of these plants compared to AN Cambará, which possibly need a more intense water deficit to activate responses of this gene, since they are already accustomed to conditions of lower water availability. However, for bZIP46, the dryland genotype presented an increase in expression under normal conditions (10\%), normalizing after $24 \mathrm{~h}$ of rehydration, suggesting that the response of this gene is rapid and occurs only in the period of greatest need. In view of this, we can observe that these responses are variable and may present a particular response depending on species and condition.

According to Borah et al. (2017), transcriptomic analysis of rice seedlings with different levels of tolerance (sensitive and tolerant) submitted to three hours of drought stress showed rapid and intense gene signaling, many of them as DREBs indicating that the transcripts are probably the initial signaling of the processes triggered by the drought. In addition, even the tolerant genotype showed high variation in gene expression over time, demonstrating the fluctuation of this variable. Therefore, to rely only on the mobility of the transcripts to predict tolerance / susceptibility becomes an error in the interpretation of the future behavior of plants against an adverse condition. In addition, Janiak et al. (2016) also observed variation in transcripts over time $(0-12 \mathrm{~h})$ and according to stress (salinity, dryness and low temperatures) in rice plants.

By observing the network in which the TFs are involved and how they are influencing other genes and metabolic activities (Figure 4), the possibility of variation in expression responses and sometimes a misinterpretation of biological responses is evident as it is based on only one or a few genes since some of them were correlated may have been influenced by the water deficit and this altered the expression of the TFs studied.

The relationship between several genes, depending on the condition and influence one exerts under another/others demonstrates dynamics and flexibility. Even in a water deficit condition, as in the case of this study, several correlated genes are involved in carotenoid synthesis routes, pathogen plant relationship and involved with lipid metabolism.

Observing the interaction network formed (Figure 4), it is also noticed that a distinct grouping involving certain TFs occurs, for example, DREB1A (Os09g0522200), DREB1B (Os09g0522000) and DREB1C (Os06g0127100). These genes are related, according to the network, with some genes for cytotoxicity-mediated cell death. It was observed that, in face of water deficit and mainly rehydration in BRS Querência genotype, these TFs had their highest expressions (Figure 3), being possibly associated to the increase of defense proteins during the period of stress and in the recovery occur the autolysis of the same, that at this moment, may no longer be necessary or remove the damaged tissues, thus ensuring the survival of the organism (Petrov et al., 2015). 
Even though it belonged to the same gene family, DREB1F (Os01g0968800) was spatially separated from the others in network analysis. Regarding its expression in this study, this gene responded more significantly to the dryland genotype (AN Cambará) under stress conditions (20\% and 10\%) (Figure 3). Observing the interaction (Figure 4) a strong association with a cytochrome P450 protein related gene (Os11g0151400) is noted. These proteins participate in the biosynthesis of sesquiterpenes, which are involved, among other functions, with the defense of the vegetable against stress conditions (Weitzel and Simonsen 2015). In a study with soybean (Glycine max L.) with different stressing conditions, it was demonstrated an increase in the expression of genes of the cytochrome P450 family in drought and that this increase was being in the plants tolerant to this condition (Yan et al., 2016). Because the expression of these TFs has been more representative in the genotype with greater tolerance to the water deficit and to be related to the genes involved in the terpene biosynthesis, we believe, as seen by other authors, that this may be a characteristic in plants with greater adaptability to this condition.

Although DREB2A (Os08g0485400) is one of the most studied TFs under drought conditions, it is related to other metabolic pathways that may or may not be altered under water deficit conditions. According to network analysis, it is associated with the metabolism of tyrosine and $\alpha$-linolenic acid, and does not interact directly with other TFs of the same family, therefore, the variations observed in the expression of this gene in our study may be associated with changes in other which can also be influenced by stress.

Interacting with several genes, bZIP23 (Os02g0766700) is directly related to ABA signaling and biosynthesis and thus closely related to dehydration (Zong et al., 2016). Expression of this gene in BRS Querência, genotype less tolerant to the imposed condition, was superior. However, it is noted that this gene is influenced by several others, being in our network the gene with the largest number of connections and because of this, the regulation of the same is highly complex. Similar situation is observed with DREBIC (Os06g0127100), which has a high correlation (demonstrated by the color intensity of the lines) with calmodulin proteins (Os01g0135700), involved with the signaling of several metabolic processes, mainly related to abiotic stresses, such as drought (Virdi et al., 2015). However, bZIP46 (Os06g0211200), bZIP72 (Os09g0456200) and DREB1E (Os02g0676800) appear to be correlated to few genes. Therefore, the study of the expression of related genes may facilitate the understanding of the signaling mechanism of these TFs in order to elucidate some responses to water stress.

Observing the genetic behavior in each studied condition, as well as the analysis of correlation/networks, we can conclude that there is no linear pattern, because it varies genotype, different humidity conditions, interaction of TFs with other genes and routes, as well as other characteristics, the response of expression and signaling is variable, as well as because we have faster responses or require a longer time to happen. Several studies have demonstrated these TF as responsive to dehydration, but we must observe the moment and the way they are activated, in this stream of dynamic responses. Another study carried out at the reproductive stage of rice plant development demonstrated contrasting responses in several genes (Auler et al., 2017) corroborating this hypothesis.

Thus, our hypothesis is confirmed in parts, demonstrating that the contrasting genotypes signal at different degrees of expression according to soil moisture; however, we cannot affirm that the flooded genotype has more significant responses in dry soil, since we observed that these are very variable, both according to the observed gene, and the 
condition. Similarly, Seeve et al. (2016) affirm that a clearer understanding of the genetic regulation of responses of plants to drought will require further studies to examine the tissue specific responses and TF under different conditions of water deficit. (Mohanty et al., 2016). Future research will restrict the focus to certain drought-sensitive TFs that function as hubs, assisting in the availability of data to map networks and thus elucidate their regulation so that strategies are plotted with the objective of assisting in drought tolerance breeding, considering all plant modules and how they react to the most critical moments of stress.

\section{ACKNOWLEDGMENTS}

The authors gratefully acknowledge the CNPq (Conselho Nacional de Desenvolvimento Científico e Tecnológico) for their financial support and the FAPERGS (Research Foundation of Rio Grande do Sul) for supporting the research, as well as the company AgroNorte/MT (Sinop - MT/Brazil BR 163, KM 852, S/N - Rural Area, 78550001) and the Dr. Ariano Martins de Magalhães Júnior (EMBRAPA TEMPERATE CLIMATE - Monte Bonito, RS/Brazil, Rodovia BR 392, km 78, $9^{\circ}$ Distrito - 96010-971) for providing seeds.

This study was financed in part by the Coordenação de Aperfeiçoamento de Pessoal de Nível Superior - Brasil (CAPES) - Finance Code 001.

\section{REFERENCES}

Agarwal M, Katiyar-Agarwal S, Zhu JK (2007) Interaction of SOS2 with nucleoside diphosphate kinase 2 and catalases reveals a point of connection between salt stress and $\mathrm{H}_{2} \mathrm{O}_{2}$ signaling in Arabidopsis thaliana. Mol Cell Bio 27:77717780

Almogueva C, Prieto-Dapena P, Díaz-Martín J, et al (2009) The HaDREB2 transcription factor enhances basal thermotolerance and longevity of seeds through functional interaction with HaHSFA9. BMC Plant Biol 9:75

Auler PA, Benitez LC, do Amaral MN, et al (2016) Evaluation of stability and validation of reference genes for RTqPCR expression studies in rice plants under water deficit. $J$ Appl Genetics 58:163-177

Auler PA, do Amaral MN, Rodrigues GDS, et al (2017) Molecular responses to recurrent drought in two contrasting rice genotypes. Planta 246(5):899-914

Bhatnagar-Mathur P, Rao JS, Vadez V, et al (2014) Transgenic peanut overexpressing the DREB1A transcription factor has higher yields under drought stress. Mol Breeding 33:327-340

Borah P, Sharma E, Kaur A, Chandel G, Mohapatra T, Kapoor S, Khurana JP (2017) Analysis of drought-responsive signaling network in two contrasting rice cultivars using transcriptome-based approach. Sci Rep 7:421-431

Cabane M, Afif D, Hawkins S (2012) Lignins and Abiotic Stress. Adv Bot Res 61:219-262

Chen JQ, Meng XP, Zhang Y, et al (2008) Over-expression of OsDREB genes lead to enhanced drought tolerance in rice. Biotechnol Lett 30:2191-2198

Dubey S, Pandey A, Sangwan R (2016) Current Developments in Biotechnology and Bioengineering: Crop Modification, Nutrition, and Food Production. Elsevier, Amsterdam

Dubouzet JG, Sakuma Y, Ito Y, et al (2003) OsDREB genes in rice, Oryza sativa L., encoded transcription activators that function in drought, high-salt and cold-responsive gene expression. Plant J 33:751-763.

Foley JA, Ramankutty N, Braumann KA, et al (2011) Solutions for a cultivated planet. Nature 478:337-342

Golldack D, Li C, Mohan H, Probst N (2014) Tolerance to drought and salt stress in plants: unraveling the signaling networks. Front Plant Sci 5:151

Gowda VRP, Henry A, Yamauchi A, et al (2011) Root biology and genetic improvement for drought avoidance in rice. Field Crops Res 122:1-13

Guimarães CM, Santos AB, Magalhães AM, Stone LF (2006) Sistemas de cultivo. In: Santos AB, Stone LF, Vieira NR (eds) A cultura do arroz no Brasil, 2nd edn. CONAB, Brasília, pp 53-96

Ito Y, Katsura K, Maruyama K, et al (2006) Functional analysis of rice DREB1/CBF-type transcription factors involved in cold-responsive gene expression in transgenic rice. Plant Cell Physiol 47:141-153

Janiak A, Kwaśniewski M, Szarejko I (2016) Gene expression regulation in roots under drought. J Exp Bot. 4:1003-1014 
Jiménez-Gómez JM (2014) Network types and their application in natural variation studies in plants. Curr Opin Plant Biol 18:80-86

Lata and Prassad (2011) Role of DREBs in regulation of abiotic stress responses in plants. $J$ Exp Bot 62:4731-4748

Liu C, Wu Y, Wang X (2012) bZIP transcription factor OsbZIP52/RISBZ5: a potential negative regulator of cold and drought stress response in rice. Planta 235:1157-1169

Livak KJ, Schmittgen TD (2001) Analysis of relative gene expression data using real-time quantitative PCR and the 2(-Delta Delta C(T)) Method. Methods 25:402-408

Lu G, Gao C, Zheng X, Han B (2009) Identification of OsbZIP72 as a positive regulator of ABA response and drought tolerance in rice. Planta 229:605-615

Mochida K, Shinozak K (2011) Advances in Omics and Bioinformatics Tools for Systems Analyses of Plant Functions. Plant Cell Physiol 52(12):2017-2038

Mohanty B, Kitazumi A, Cheung CYM, et al (2016) Indentification of candidate network hubs involved in metabolic adjustments of rice under drought stress by integrating transcriptome data and genomce-scale metabolic network. Plant Sci 242:224-239.

Nijhawan A, Jain M, Tyagi AK, Khurana JP (2008) Genomic survey and gene expression analysis of the basic leucine zipper transcription factor family in rice. Plant Physiol 146:333-350.

Oh SJ, Song SI, Kim YS, Jang et al (2005) Arabidopsis CBF3/DREB1A and ABF3 in transgenic rice increased tolerance to abiotic stress without stunting growth. Plant Physiol 138:341-351.

Pandey, V., and Shukla, A. (2015) Acclimation and tolerance strategies of rice under drought stress. Rice Sci. 22, $147-$ 161.

Petrov V, Hille J, Mueller-Roeber B, Gechev TS (2015) ROS-mediated abiotic stress-induced programmed cell death in plants. Front Plant Sci 6:69.

Ptashne M (1988) How eukaryotic transcriptiona1 activators work. Nature 335(6192):683-689

Qin F, Sakuma Y, Tran LS, et al (2008) Arabidopsis DREB2A-interacting proteins function as RING E3 ligases and negatively regulate plant drought stress-responsive gene expression. Plant Cell 20(6):1693-1707

RCore Team (2013) R: a Language and Environment for Statistical Computing. R Foundation for Statistical Computing, Vienna, Austria

Reddy GL, Reddy AR (2008) Rice DREB1B promoter shows distinct stress-specific responses, and the overexpression of cDNA in tobacco confers improved abiotic and biotic stress tolerance. Plant Mol Biol 68(6):533-555

Riechmann JL, Heard J, Martin G, et al (2000) Arabidopsis transcription factors: genome-wide comparative analysis among eukaryotes. Science 290(5499):2105-2110

Sakuma Y (2006a) Dual function of an Arabidopsis transcription factor DREB2A in water-stress-responsive and heatstress-responsive gene expression. PNAS 103(49): 18822-18827

Sakuma Y (2006b) Functional Analysis of an Arabidopsis Transcription Factor, DREB2A, Involved in DroughtResponsive Gene Expression. Plant Cell 18(5):1292-1309

Sakuma Y, Liu Q, Dubouzet JG, et al (2002) DNA- binding specificity of the ERF/AP2 domain of Arabidopsis DREB'S, transcription factors involved in dehydration and cold-inducible gene expression. Biochem Biophys Res Commun 290:998-1009

Sato Y, Antonio BA, Namiki N, et al (2011) RiceXPro: a platform for monitoring gene expression in japonica rice grown under natural field conditions. Nucleic Acids Res 39:D1141-D1148

Sato Y, Namiki N, Takehisa H, et al (2013) Rice FREND: a platform for retrieving coexpressed gene networks in rice. Nucleic Acids Res 41:D1214-D1221

Seeve CM, Cho IJ, Hearne LB, et al (2017) Water-deficit-induced changes in transcription factor expression in maize seedlings. Plant Cell Environ 40(5):686-701. https://doi.org/10.1111/pce.12891

Serin EAR, Nijveen H, Hilhorst HWM, Ligterink W (2016) Learning from co-expression networks: possibilities and challenges. Front Plant Sci 7:444

Serraj R, Mcnally KL, Slamet-Loedin I, et al (2011) Drought resistance improvement in rice: An integrated genetic and resource management strategy. Plant prod sci 14:1-14

Shi B, Ni L, Zhang A, et al (2012) OsDMI3 Is a Novel Component of Abscisic Acid Signaling in the Induction of Antioxidant in Leaves of Rice. Mol Plant 5(6):1359-1374

Silva AT, Ribone PA, Chan RL, et al (2016) A Predictive Coexpression Network Identifies Novel Genes Controlling the Seed-to-Seedling Phase Transition in Arabidopsis thaliana. Plant Physiol 170(4):2218-2231

Smart RE, Bingham GE (1974) Rapid Estimates of Relative Water Content. Plant Physiol 53(2):258-260.

Tang N, Zhang H, Li X, et al (2012) Constitutive activation of transcription factor OsbZIP46 improves drought tolerance in rice. Plant Physiol 158(4):1755-1768

Terashima A, Takumi S (2009) Allopolyploidization reduces alternative splicing efficiency for transcripts of the wheat DREB2 homolog, WDREB2. Genome 52:100-105

Thomashow MF (1999) Plant cold acclimation: freezing tolerance genes and regulatory mechanisms. Annu Rev Plant Physiol Plant Mol Biol 50:571-599

Usadel B, Obayashi T, Mutwil M, et al (2009) Co-expression tools for plant biology: opportunities for hypothesis generation and caveats. Plant Cell Environ. 32:1633-1651 
Virdi AS, Singh S, Singh P (2015) Abiotic stress responses in plants: roles of calmodulin-regulated proteins. Front Plant Sci 6:809

Wei T, Deng K, Liu D, et al (2016) Ectopic expression of DREB transcription factor, AtDREB1A, confers tolerance to drought in transgenic Salvia miltiorrhiza. Plant Cell Physiol 57(8):1593-1609

Weitzel C, Simonsen HT (2015) Cytochrome P450-enzymes involved in the biosynthesis of mono- and sesquiterpenes. Phytochem Rev 14:7-24

Xiang Y, Tang N, Du H, et al (2008) Characterization of OsbZIP23 as a key player of the basic leucine zipper transcription factor family for conferring abscisic acid sensitivity and salinity and drought tolerance in rice. Plant Physiol 148:1938-1952

Yamaguchi-Shinozaki K, Shinozaki K (1993) The plant hormone abscisic acid mediates the drought-induced expression but not the seed-specific expression of rd22, a gene responsive to dehydration-stress in Arabidopsis thaliana. Mol Gen Genet 238:17-25

Yamaguchi-Shinozaki K, Shinozaki K (2006) Transcriptional regulatory networks in cellular responses and tolerance to dehydration and cold stresses. Anпи Rev Plant Biol 57:781-803

Yan Q, Cui X, Lin S, et al (2016) GmCYP82A3, a Soybean Cytochrome P450 Family Gene Involved in the Jasmonic Acid and Ethylene Signaling Pathway, Enhances Plant Resistance to Biotic and Abiotic Stresses. PLoS ONE 11(9):e0162253

Zhu JK (2002) Salt and drought stress signal transduction in plants. Annu Rev Plant Biol 53:247-273

Zong W, Tang N, Yang J, et al (2016) Feedback regulation of ABA signaling and biosynthesis by a bZIP transcription factor targets drought-resistance-related genes. Plant Physiol 171:2810-2825 